\title{
Silencing of antiapoptotic survivin gene by multiple approaches of RNA interference technology
}

\author{
Xiang Ling and Fengzhi Li
}

BioTechniques 36:450-460 (March 2004)

\begin{abstract}
Silencing of mammalian gene expression by RNA interference (RNAi) technology can be achieved using small interfering RNA (siRNA) or short hairpin RNA (shRNA). However, the relative effectiveness of these two approaches is not known. It is also not clear whether gene-specific shRNA transcribed from an RNA polymerase II (Pol II)-directed promoter in a fusion form can disrupt the targeted gene expression. Here, we report that using both luciferase and antiapoptotic survivin genes as targets, both siRNA and shRNA approaches significantly silenced the targeted gene expression in cancer cells. We further demonstrated that shRNAs transcribed from an RNA Pol II-mediated promoter in a green fluorescent protein (GFP) fusion form at the 3'-untranslated region silenced luciferase and survivin expression as well, suggesting that the extra RNA sequence outside of the shRNA hairpin does not disrupt shRNA function. We also showed that silencing of survivin expression selectively induces apoptosis in transfected cells. Together, we have validated multiple approaches of RNAi technology using both survivin and luciferase genes as targets and demonstrated for the first time that GFP-shRNAs transcribed from an RNA Pol II-mediated promoter could mediate gene silencing, which may lead to new directions for the application of RNAi technology.
\end{abstract}

\section{INTRODUCTION}

RNA interference (RNAi) or posttranscriptional gene silencing (PTGS) is an evolutionarily conserved biological response to double-stranded RNA (dsRNA) for degradation of the sequence-specific homologous mRNA. Biochemical reactions that recapitulate this phenomenon generate dsRNA fragments of 21-23 nucleotides, known as small interfering RNA (siRNA), from the long dsRNA (1-4). This conserved mechanism in mammalian cells is used to mediate resistance to both endogenous parasitic and exogenous pathogenic nucleic acids and regulate the expression of protein-coding genes (3). Although the use of siRNAs to silence genes in vertebrate cells was only reported recently, the emerging literature indicates that most vertebrate genes can be silenced using this technology (5). Currently, RNAi technology is extremely attractive, since it is a time-effective means to manipulate gene expression experimentally and to probe gene function even on a wholegenome scale $(1,2,4)$.

Using a Drosophila in vitro system, it was demonstrated that long dsRNA could be processed to 21-23 nucleotides of siRNA, which guides the degradation of mRNA within the region of identity with the siRNA $(6,7)$. However, application of long dsRNA to silence protein-coding genes in mammalian cells has potential impediments. It has not been possible to detect specific RNAi in commonly used mammalian cell culture systems $(8,9)$. Long dsRNA can trigger nonspecific antiviral response to induce the production of interferon synthesis in mammalian cells $(10,11)$. However, it was demonstrated that chemically synthesized siRNAs induce gene silencing in a wide range of human and mouse cell lines in culture $(12,13)$. On the other hand, an approximately 70-nucleotide hairpin precursor RNA transcribed from the Let-7 gene $(14,15)$ can be posttranscriptionally processed into a mature approximately 21-nucleotide
siRNA to induce mRNA-specific gene silencing in Caenorhabditis elegans (14) and in human cells (15). Consistent with this observation, it was shown that chemically synthesized short hairpin RNA (shRNA) induces sequence-specific gene silencing in mammalian cells (16), and the in vitro transcribed shRNA or expression vector-based shRNA does the same $(16,17)$. However, the relative effectiveness of these different approaches of RNAi technology in mammalian gene silencing is not clear. Moreover, it is not known whether the shRNA generated from an RNA polymerase II (Pol II) promoter-driven vector could silence the targeted gene.

Survivin is a novel member of the inhibitor of apoptosis (IAP) protein family. It is undetectable in most normal adult tissues but highly expressed in cancer, and the expression of survivin is associated with carcinogenesis, cancer progression, poor prognosis, drug resistance, and short patient survival (18). Although 
it has been shown that inhibition of survivin expression and/or function by survivin antisense or dominantnegative mutants triggers apoptosis (19-23) and cell division defects $(24,25)$, silencing of survivin gene using multiple approaches of RNAi technology has not been studied and may be a more effective means for cancer treatment. In this paper, we employed both exogenous luciferase gene and endogenous survivin gene as targets to evaluate the effect of different approaches of RNAi technology on sequence-specific gene silencing and cellular responses.

\section{MATERIALS AND METHODS}

\section{Cell Culture and Reagents}

HeLa cells were maintained in Dulbecco's modified Eagle's medium (DMEM) supplemented with $10 \%$ serum (Cellgro, Herndon, VA, USA) and $100 \mathrm{U} / \mathrm{mL}$ of penicillin/ 0.1 $\mu \mathrm{g} / \mathrm{mL}$ of streptomycin (Invitrogen, Carlsbad, CA, USA) in a $5 \% \mathrm{CO}_{2}$ incubator at $37^{\circ} \mathrm{C}$. Anti-survivin antibody (FL-142) was purchased from Santa Cruz Biotechnology (Santa Cruz, CA, USA). Phenazine methosulfate, 2,3-bis (2-methoxy-4nitro-5-sulfophenyl)-2 $\mathrm{H}$-tetrazolium5-carboxanilide (XTT), monoclonal anti-actin antibody, and goat peroxidase-conjugated anti-rabbit immunoglobulin $\mathrm{G}$ ( $\mathrm{IgG}$ ) antibody were purchased from Sigma (St. Louis, MO, USA). Dual-Luciferase Reporter Assay system was purchased from Promega (Madison, WI, USA). Oligotransfectamine ${ }^{\mathrm{TM}}$ reagent and Lipofectamine ${ }^{\mathrm{TM}} 2000$ reagents were purchased from Invitrogen.

\section{siRNA Preparation}

The six human survivin mRNAspecific RNA oligonucleotides with 3'-TT overhangs were chemically synthesized (Xeragon, Huntsville, AL, USA) (Figure 1A). The corresponding forward RNA oligonucleotides were labeled by 6-FAM fluorescence dyes at the $3^{\prime}$ end $(*)$ (Figure 1A) and purified by highperformance liquid chromatography
(HPLC). Equal moles of forward and reverse RNA oligonucleotides were mixed together to a final concentration of $20 \mu \mathrm{M}$ in annealing buffer (100 $\mathrm{mM}$ potassium acetate, $30 \mathrm{mM}$ HEPES-KOH, $2 \mathrm{mM}$ magnesium acetate, $\mathrm{pH}$ 7.4), respectively. After denaturation at $90^{\circ} \mathrm{C}$ for $1 \mathrm{~min}$, each mixture (Sri-2, invSRi-2, and Sri-3; Figure 1A) was annealed at $37^{\circ} \mathrm{C}$ for $60 \mathrm{~min}$ and stored at $-80^{\circ} \mathrm{C}$ for transfection experiments. A scramble RNA duplex (designated scraSRi) was also prepared, the same as above, for an extra negative control in this study (Figure 1A). The scramble sequence was not present in mammalian cells by Basic Local Alignment Search Tool (BLAST) search at the National Center for Biotechnology Information $(\mathrm{NCBI})$.

\section{Molecular Cloning of H1 RNA Promoter}

On the basis of human H1 RNA gene sequence $\left(\right.$ GenBank $^{\circledR}$ accession no. X16612), we synthesized two primers, hH1RNA5'P2 (5'-ACG CGTCGAC GAA CGC TGA CGT CAT CA-3', SalI underlined) and hH1RNA3'P1 (5'-CGGGATCC GGG AAA GAG TGG TCT CAT-3', BamHI underlined), and PCR-amplified a 223-bp human H1 RNA promoter sequence using human genomic DNA as a template. The PCR product was gel-purified, digested with SalI and BamHI, and cloned into upstream of luciferase gene in the pLuc reporter vector at the SalI and BamHI sites to generate a new vector designated pH1Luc. The promoter sequence in pH1Luc was confirmed by sequencing, and its function was tested by luciferase activity assay.

\section{Construction of Plasmids Expressing Various shRNA}

Two wild-types and one mutant of survivin shRNA gene were constructed to target survivin mRNA. The forward (F) and reverse (R) chains of each survivin shRNA gene were chemically synthesized (Integrated DNA Technologies, Coralville, IA, USA). Each shRNA gene was flanked with $B a m \mathrm{HI}$ at $5^{\prime}$ end and EcoRI at $3^{\prime}$ end 
(underlined) for a cloning purpose. The DNA sequences of these shRNA genes are shRNA1-F (5'-CGGGATCC TGA GGC TGG CTT CAT CCA CTG CTT CAA GAG CGC AGT GGA TGA AGC CAG CCT CTT TTT GTC GAC GAATTCC-3') and shRNA1-R (5'-GGAATTC GTC GAC AAA AAG AGG CTG GCT TCA TCC ACT GCG CTC TTG AAG CAG TGG ATG AAG CCA GCC TCA GGATC CCG-3') for shRNA1 gene; shRNA1L-F (5'-CGGGATCC TGA GGC TGG CTT CAT CCA CTG CCC TTC AAG AGC GGG CAG TGG ATG AAG CCA GCC TCT TTT TGT CGA CGAATTCC-3') and shRNA1L-R (5'-GGAATTC GTC GAC AAA AAG AGG CTG GCT TCA TCC ACT GCC CGC TCT TGA AGG GCA GTG GAT GAA GCC AGC CTC AGGATCCCG-3') for shRNA1L gene; and shRNA1m-F (5'-CGGGATCC TGA GGC CAG CTG TAT CCC TTG CTT CAA GAG CGC AAG GGA TAC AGC TGG CCT CTT TTT GTC GAC GAATTCC-3') and shRNA1m-R (5'-GGAATTC GTC GAC AAA AAG AGG CCA GCT GTA TCC CTT GCG CTC TTG AAG CAA GGG ATA CAG CTG GCC TCA GGATCCCG-3') for shRNA1m gene (mutant shRNA1 gene). One wild-type and one mutant of luciferase shRNA gene were also chemically synthesized with the same design above for a cloning purpose. The DNA sequences are lucshRNA1F (5'-CGGGATCC TGA AGA GAT ACG CCC TGG TTC CTT TCA AGA
GCA GGA ACC AGG GCG TAT CTC TTC TTT TTG TCG ACGAATTCC$\left.3^{\prime}\right)$ and lucshRNA1-R (5'-GGAATTC GTC GAC AAA AAG AAG AGA TAC GCC CTG GTT CCT GCT CTT GAA AGG AAC CAG GGC GTA TCT CTT CAGGATCCCG-3') for lucshRNA1 gene; and lucshRNA1m-F (5'CGGGATCC TGA AGA GGC ACG CCT CGG TTC CTT TCA AGA GCA GGA ACC GAG GCG TGC CTC TTC TTT TTG TCG ACGAATTCC-3') and lucshRNA1m-R (5'-GGAATTC GTC GAC AAA AAG AAG AGG CAC GCC TCG GTT CCT GCT CTT GAA AGG AAC CGA GGC GTG CCT CTT CAGGATCCCG-3') for lucshRNA1m gene (mutant lucshRNA1 gene). After the complementary sequences were annealed and digested with BamHI and EcoRI, each corresponding shRNA gene was either cloned into the pEGFPc1 vector (BD Biosciences Clontech, Palo Alto, CA, USA) at the BglII and EcoRI sites (the new vectors were designated as pG-shRNA1, pG-shRNA1L, pG-shRNA1m, pG-lucshRNA1, and pG-lucishRNA1m) or replaced the luciferase gene at the BamHI and EcoRI sites in the pH1Luc vector cloned above (the new vectors were designated as pH1-shRNA1, pH1-shRNA1L, pH1shRNA1m, pH1-lucshRNA1, and pH1lucishRNA1m). The shRNA genes were confirmed by DNA sequencing. The bold TGA sequence is an in-frame stop codon. Downstream of TGA is an untranslated region when the shRNA gene is fused to the green fluorescent protein (GFP) expression vector.

\section{Transfection of siRNA or shRNA Expression Vectors}

One day prior to transfection, $3 \times 10^{5}$ cells/well were seeded in 6-well plates containing culture medium without antibiotics. HeLa cells at $30 \%-50 \%$ confluence were transfected as follows. Serum-free DMEM $(175 \mu \mathrm{L})$ containing $60 \mathrm{nM}$ siRNA was mixed with $15 \mu \mathrm{L}$ serum-free DMEM containing $6 \mu \mathrm{L}$ Oligofectamine reagents and kept at room temperature. After the medium in the 6-well plate was replaced by serum-free DMEM $(800 \mu \mathrm{L} /$ well $)$, the siRNA/oligofectamine mixture $(190 \mu \mathrm{L})$ prepared above was evenly added into each well within 20-45 min after the mixture was prepared. The plate was incubated at $37^{\circ} \mathrm{C}$ for $4 \mathrm{~h}$, and then $500 \mu \mathrm{L}$ DMEM containing 30\% fetal bovine serum (FBS) were added into each well. Cells were analyzed $48 \mathrm{~h}$ after transfection by Western blot analysis or DAPI $\left(4^{\prime}, 6\right.$ diamidino-2-phenylindole) staining/ immunofluorescence microscopy. Alternatively, cells (2000/well) were reseeded into 96-well plates for the XTT assay $16 \mathrm{~h}$ after transfection.

Transfection of cells with various shRNA vectors and/or reporter vectors was performed using Lipofectamine 2000 reagent. HeLa cells
A GCGCCUGCACCCCGGAGCGT*
SRi-2
TTCGCGGACGUGGGGCCUCGC

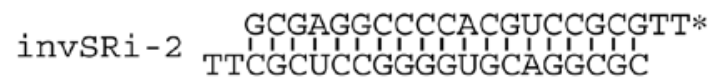

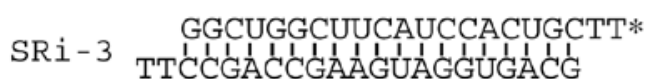

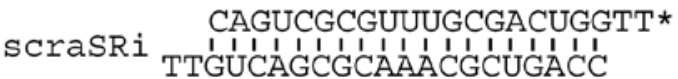

B

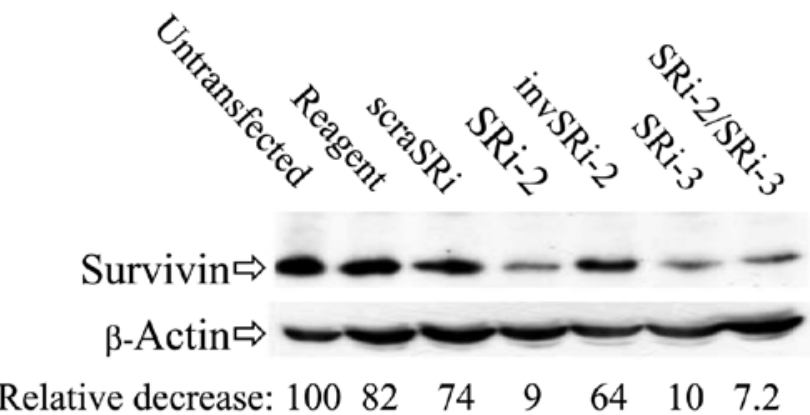

Figure 1. Silencing of survivin expression by chemically synthesized siRNAs. (A) Structures of siRNAs utilized in this study. SRi- 2 and SRi-3 are two survivin mRNA-specific siRNAs. InvSRi-2 (a reverse sequence of SRi-2) and scraSRi (a scramble siRNA) are two negative controls. (B) Down-regulation of survivin expression by siRNAs. HeLa cells were transfected with various siRNAs. Cells were lysed $48 \mathrm{~h}$ after transfection, and survivin expression was determined by Western blot analysis. The relative percentage of survivin decrease after normalization to $\beta$-actin internal control is indicated. siRNA, small interfering RNA; *, indicates $3^{\prime}$ end of forward RNA oligonucleotide labeled with 6-FAM fluorescent dye. 
were seeded in 24 -well $\left(5 \times 10^{4} /\right.$ well for luciferase assay) and 6-well $(5 \times$ $10^{5} /$ well for Western blot analysis) plates. Cells at 70\%-80\% confluence were transfected following the manufacturer's protocol. Briefly, $0.6 \mu \mathrm{g}$ of vector DNA in $50 \mu \mathrm{L}$ serum-free DMEM was mixed with up to $1.5 \mu \mathrm{L}$ Lipofectamine 2000 reagent in $50 \mu \mathrm{L}$ serum-free DMEM for each well (24-well plate). Three micrograms of vector DNA in 100 $\mu \mathrm{L}$ serum-free DMEM were mixed with $5 \mu \mathrm{L}$ Lipofectamine 2000 in 100 $\mu \mathrm{L}$ serum-free DMEM for each well (6-well plate). In other experiments, different amounts of relevant vector DNA $(2,1,0.5$, and $0.1 \mu \mathrm{g})$ were used for transfection in each well (12-well plate) for detection of dose-depen- dent effect, while the same amount of pRL-TK internal control vectors (40 ng) was used for each well. After incubation of the DNA/Lipofectamine 2000 mixture at room temperature for 20-30 $\mathrm{min}$, the mixture was added to each well containing complete culture medium $(500 \mu \mathrm{L} /$ well for 24-well plates and $2000 \mu \mathrm{L} /$ well for 6 -well plates). For testing the dosedependent effect, cells were lysed at $48 \mathrm{~h}$ after transfection. For exploring the time-dependent effect, cells were harvested at 16, 24, 48, and $72 \mathrm{~h}$ after transfection.

\section{Western Blot Analysis}

Cells were washed with phosphatebuffered saline (PBS) and lysed at $4^{\circ} \mathrm{C}$ for 30 min in PBS containing $1 \%$ Nonidet ${ }^{\circledR}$ P-40, $0.5 \%$ sodium deoxycholate, $0.1 \%$ sodium dodecyl sulfate (SDS), $10 \mu \mathrm{g} / \mathrm{mL}$ phenylmethyl sulfonyl fluoride, and $20 \mu \mathrm{M}$ leupeptin. Cell extracts were cleared by centrifugation at $15,000 \times \mathrm{g}$ for $20 \mathrm{~min}$ at $4^{\circ} \mathrm{C}$, and the supernatant was used for Western blot analyses. Up to $75 \mu \mathrm{g}$ of total protein from each sample was heated at $95^{\circ} \mathrm{C}$ for $5 \mathrm{~min}$ after mixing with an equal volume of $2 \times$ SDS loading buffer. Samples were separated on $15 \%$ SDS-polyacrylamide gel electrophoresis (SDS-PAGE) and electrotransferred to Immobilon ${ }^{\text {TM }}$-P membranes (Millipore, Billerica, MA, USA). The membrane was blocked in $5 \%$ skimmed milk in TBST buffer $(20 \mathrm{mM}$ Tris-HCl, $\mathrm{pH}$ 7.5, 0.137

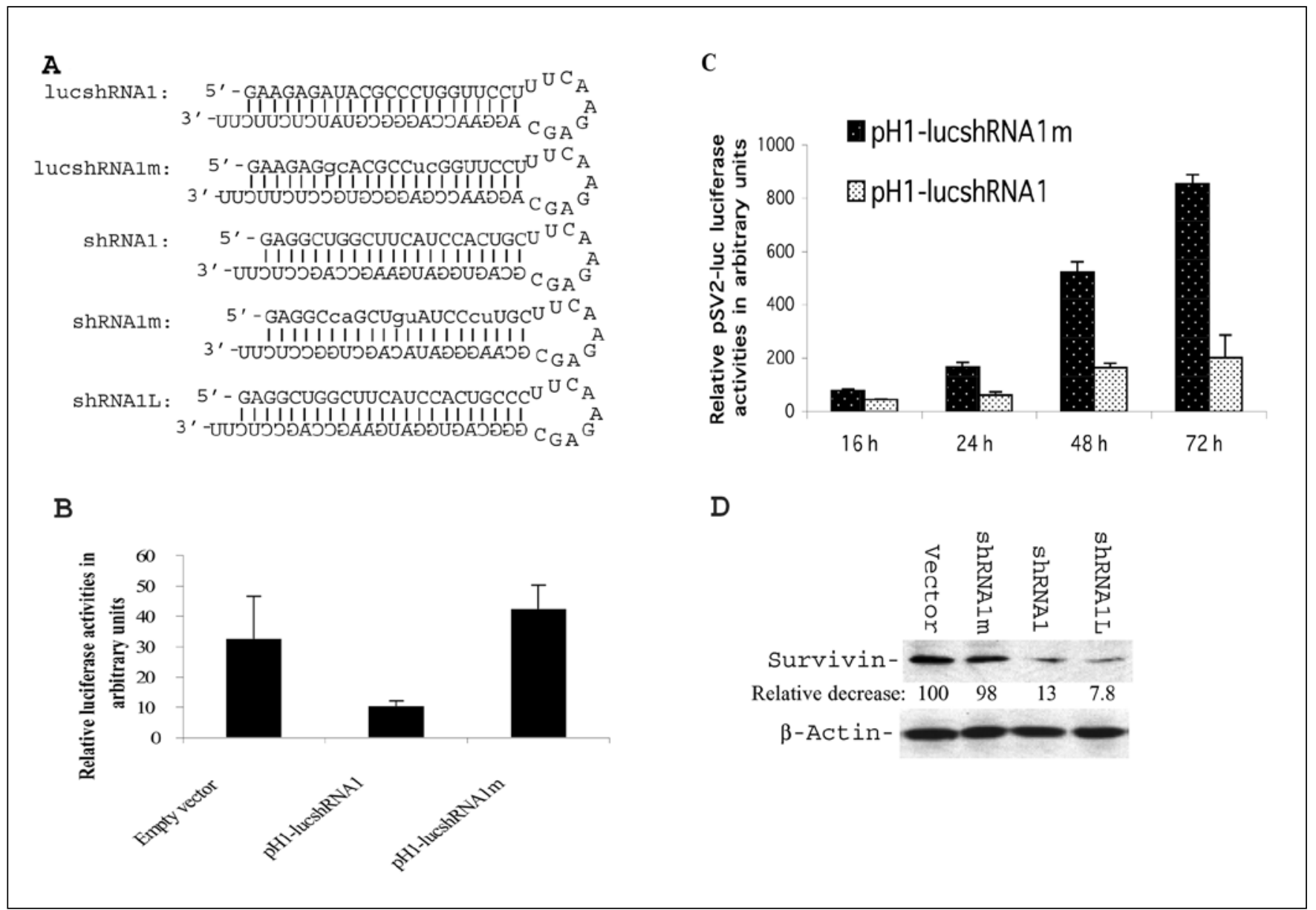

Figure 2. Silencing of luciferase and survivin genes by H1 RNA promoter-transcribed shRNAs. (A) Structures of shRNAs transcribed from H1 RNA promoter vectors. LucshRNA1 and lucshRNA1m (mutant) target luciferase mRNA. ShRNA1 (21 nucleotides), shRNA1m (mutant), and shRNA1L (23 nucleotides) target survivin mRNA. (B) Silencing of luciferase gene by lucshRNA1. Luciferase activities were measured $48 \mathrm{~h}$ after transfection. (C) Sustained inhibitory effects of lucsiRNA1 on luciferase activity. Representative experiments in triplicate are shown as the mean ( \pm SD). (D) Silencing of survivin by survivin shRNAs. Survivin expression was determined by Western blot analysis $48 \mathrm{~h}$ after transfection. The relative percentage of survivin decrease after normalization to $\beta$-actin is indicated. shRNA, short hairpin RNA. 
$\mathrm{M} \mathrm{NaCl}$, and $0.05 \%$ Tween ${ }^{\circledR} 20$ ) at room temperature for 2 to $3 \mathrm{~h}$, and then incubated overnight at $4^{\circ} \mathrm{C}$ in TBST buffer containing 5\% BSA and survivin polyclonal antibody (1:500) from rabbit with a gentle shaking. After washing with TBST, the membrane was incubated in 5\% skimmed milk in TBST buffer containing goat anti-rabbit $\operatorname{IgG}$ (1:5000), for 45-60 min at room temperature. Survivin proteins were detected by an electrochemiluminescence protein detection kit (ECL ${ }^{\mathrm{TM}}$; Amersham Biosciences, Piscataway, NJ, USA) and visualized by autoradiography. For normalization of protein loading, the same membranes were stripped by stripping buffer (100 mM 2-mercaptoethanol, 2\% SDS, $62.5 \mathrm{mM}$ Tris-HCl, $\mathrm{pH}$ 6.7) and used for Western blot analysis with a monoclonal antibody against $\beta$-actin at a dilution of $1: 1000$ by the same procedure.

\section{Luciferase Reporter Assay}

The Dual-Luciferase Reporter Assay system was used for the luciferase assay. Cells were transfected with the relevant siRNA and/or expression vectors in 24-well plates as described above. The transfected cells were washed with PBS and lysed at the specified time points with $1 \times$ passive lysis buffer provided in the kit (up to $200 \mu \mathrm{L} /$ well) on a shaker for up to 30 $\mathrm{min}$ at room temperature. Twenty microliters of cell lysates/well were used to measure the Firefly and Renilla luciferase activity in a Luminometer by subsequently adding $20 \mu \mathrm{L}$ luciferase assay reagent and $20 \mu \mathrm{L}$ Stop \& $\mathrm{Glo}^{\circledR}$ reagent provided in the kit from Promega. The data were normalized to Renilla luciferase activity (internal control) as arbitrary units and plotted as a histogram.

\section{XTT Assay}

This assay is based on the principle that viable cells reduce yellow tetrazolium salt, XTT, to an orange formazan dye in the presence of phenazine methosulfate. Sixteen hours after transfection as described above, cells (2000 cells in $100 \mu \mathrm{L} /$ well) were reseeded in 96-well plates
(Corning Costar, Acton, MA, USA). The XTT assay was performed at $72 \mathrm{~h}$ after transfection. That is, 50 $\mu \mathrm{L} /$ well of XTT solution $(1 \mathrm{mg} / \mathrm{mL}$, freshly prepared) in DMEM containing phenazine methosulfate $(25 \mu \mathrm{M})$ were added to each well in the 96well plate. After cells were continuously incubated in the $5 \% \mathrm{CO}_{2}$ incubator at $37^{\circ} \mathrm{C}$ for $2 \mathrm{~h}$, the absorbance (A) of cells was measured at $490 \mathrm{~nm}$ using an Ultra Microplate Reader (Bio-Tek Instruments, Winooski, VT, USA). The viable cell numbers were calculated from the standard curve of cell number to A values (OD) at $490 \mathrm{~nm}$.

\section{Immunofluorescence Microscopy}

Cells were seeded on the round glass coverslips coated with $2 \%$ gelatin (Sigma) in 12-well plates. Cells at about $40 \%$ confluence were transfected with the relevant siRNA as described above. Forty-eight hours after transfection, cells were washed once with PBS and fixed overnight with $4 \%$ paraformaldehyde in PBS at $4^{\circ} \mathrm{C}$. The fixed cells on the round coverslips were permeabilized with PBS containing 2\% BSA and $0.2 \%$ Triton X-100. The nuclei were stained with DAPI $(0.5 \mu \mathrm{g} / \mathrm{mL})$ in PBS for 10 min. The coverslips with cells were mounted on glass slides using Gel/ Mount ${ }^{\mathrm{TM}}$ solution (Biomedia, Foster City, CA, USA). Cell images were captured under a Zeiss Axiovert 100M digital fluorescence microscope (Carl Zeiss MicroImaging, Thornwood, NY, USA) and processed using Photoshop ${ }^{\circledR}$ software.

\section{RESULTS}

\section{Down-Regulation of Survivin Expression by Chemically Synthesized siRNA}

The siRNAs utilized in this study are shown in Figure 1A. SRi-2 and SRi-3 target survivin mRNAs are in exon 1 and exon 2, respectively, and invSRi-2 is the reverse sequence of SRi-2 for a negative control, and scraSRi is a scramble siRNA control. To study the effect of these siRNAs on 
survivin expression, HeLa cells were transfected with and without these siRNAs separately and in combination (Figure 1B). Cells were lysed $48 \mathrm{~h}$ after transfection, and survivin expression was determined by Western blot analysis. As shown in Figure 1B, SRi-2 and SRi-3 significantly decreased survivin expression either separately or in combination, while survivin expression was largely unchanged in various controls (Figure 1B).

\section{Inhibition of Survivin and Luciferase Expression by H1 RNA Promoter-Mediated Expression of ShRNA Genes}

It was reported that while long dsRNA could not be efficiently processed into 21-25 nucleotides siRNA in mammalian cells $(8,9)$, shRNA can be processed into siRNA to silence the targeted gene $(16,17)$. In this context, we constructed a number of H1 RNA promoter-mediated shRNA expression vectors to target either luciferase or survivin mRNA. Figure $2 \mathrm{~A}$ shows the structures of these shRNAs transcribed from the H1 RNA promoterdriven vectors. LucshRNA1 and lucshRNA1m (mutant) were designed to target luciferase mRNA, while shRNA1 (21 nucleotides), shRNA1L (23 nucleotides), and shRNA1m (mutant) to target survivin mRNA. To test whether these shRNAs work in mammalian cells, the pSV2-luc reporter vector was cotransfected into HeLa cells with vectors for lucshRNA1 and lucshRNA1m or the empty vector, respectively, and luciferase activities were measured. As shown, luciferase-specific shRNA, but not mutant shRNA, inhibited luciferase activities (Figure 2B), and the inhibition of luciferase activity by shRNAs appears to be a sustained event within the effective time of transient transfection (Figure 2C). The shRNA in this system only showed a mild dose-dependent event in the range tested (0.1-2 $\mu \mathrm{g}$, not shown). Next, survivin shRNA vectors were transfected into HeLa cells, and survivin expression was determined by Western blot analysis. The results showed that this approach inhibited survivin expression as well (Figure 2D).

\section{Inhibition of Luciferase and Survivin Expression by an RNA Pol II Promoter-Mediated Expression of the GFP-Fused shRNA Genes}

Since we (in this paper) and others $(16,17)$ have shown that H1 RNA promoter-mediated or U6 RNA promoter-mediated expression of shRNA can silence gene expression, we intended to test whether an RNA Pol II promoter-transcribed shRNA in a fusion form could silence gene expression. shRNA genes were inserted into the pEGFPc1 vector at the $3^{\prime}$ end of GFP cDNA (refer to Materials and Methods). Figure 3A shows the structures of shRNAs transcribed from these vectors. The pG-lucshRNA1 and pGlucshRNA1m vectors were cotransfected with the pSV2-luc reporter vector into HeLa cells, respectively, to test the silencing of luciferase activity, or pG-shRNA1, pG-shRNA1m and $\mathrm{pG}$-shRNA1L vectors were transfected into HeLa cells, respectively, to test survivin expression silencing. These experiments revealed that shRNAs transcribed from the RNA Pol II promoter in a GFP-fused form inhibited luciferase (Figure 3B) and survivin (Figure $3 \mathrm{C}$ ) expression.

\section{Inhibition of Cell Growth by Targeting Survivin with Different Approaches of RNAi Technology}

Next, we investigated the cellular responses to RNAi-mediated survivin expression silencing. HeLa cells were transfected with survivin siRNAs or G-shRNA vectors, and viable cell numbers were measured 3 days after transfection by XTT assay. Consistent with the fact that survivin siRNA or GshRNA inhibited survivin expression (Figures 1B and 3C), transfection of survivin siRNAs or G-shRNA vectors resulted in a significant decrease in viable cell numbers (Figure 4, A and B).

\section{Induction of Apoptosis by siRNA Silencing of Survivin}

Since the transient transfection efficiency in our experiments was about $20 \%-60 \%$ of total cells, the decrease in viable cell numbers by 
survivin siRNAs or shRNA in the XTT assay may reflect apoptosis in the transfected cells. To examine this possibility, we investigated cell morphological changes after siRNA transfection. DAPI staining and fluorescence microscopy revealed apoptotic morphologies (condensed small nuclei) (Figure 5). More than 60\% of transfected cells showed apoptosis statistically (not shown).

\section{DISCUSSION}

It was reported that both chemically synthesized siRNA $(12,13)$ and U6 promoter-mediated or $\mathrm{H} 1$ RNA promoter-mediated expression of shRNA gene in mammalian cells $(16,17)$ silence genes expression. It has not been reported whether RNA Pol II promoter-mediated expression of shRNA gene in a fusion form could silence gene expression. In this report, we utilized multiple approaches of RNAi technology, including chemically synthesized siRNAs, the H1 RNA promoter-controlled expression of shRNAs, and the RNA Pol II promoter-mediated expression of shRNAs in a GFP-fused form to target both the endogenous antiapoptotic survivin gene and the exogenous luciferase reporter gene to evaluate the effectiveness of different RNAi approaches on gene silencing. We found that all of these approaches significantly silence targeted gene expression. The observation that the RNA Pol II promoter-mediated expression of shRNA in a GFP-fused form silenced targeted gene expression, suggests that the extra RNA sequence outside of the shRNA loop does not disrupt shRNA function. To our knowledge, this is the first evidence that shRNA could function in a fusing form in an untranslated region. This finding may lead to new directions for the application of RNAi technology and provide the feasibility that many superior RNA Pol II-mediated mammalian expression vectors could be utilized to drive the corresponding shRNA gene expression to silence the targeted gene expression. For example, an inducible Pol II-mediated expression vector such as the Tet-On or Tet-Off system may be used to control the expression of shRNA in mammalian cells. This will be critical for functional analysis of cell viability-essential genes. In addition, this approach is a cost- and time-effective alternative way to study the function of a targeted gene in both cell-based systems and transgenic animals. The demonstration of the effectiveness of RNA Pol II-mediated expression of shRNA in GFP-fused form will provide an additional advantage that the transfected cells are easily monitored during experiments.

When evaluating the relative effectiveness of different RNAi approaches on gene silencing, we must consider transfection efficiencies for different approaches of RNAi technology in given cell types. This is because effectiveness of cellular responses
Figure 3. Silencing of survivin and luciferase genes by green fluorescent protein (GFP)-fused shRNAs. (A) Structures of G-shRNAs (translation stop codons were bold). (B) Silencing of luciferase gene by G-lucshRNA1. The transfection and luciferase assay are as in Figure 2. A representative experiment in triplicate is shown as the mean ( \pm SD). (C) Silencing of survivin by G-shRNAs. Survivin expression was determined by Western blot analysis $48 \mathrm{~h}$ after transfection. The relative percentage of survivin decrease after normalization to $\beta$-actin is indicated. shRNA, short hairpin RNA. 
to different approaches of RNAi-mediated gene silencing is closely associated with the transfection efficiency. In our experimental system, the synthesized siRNA was more easily transfected into most cancer cell lines than the shRNA vectors (not shown). Therefore, the synthesized siRNA apparently was more effective overall in silencing the targeted gene. However, vector-based expression of shRNA could be used for generation of stable cell lines, which is desirable for gene function studies. Alternatively, the siRNA approach is likely more effective than the shRNA approach, since mammalian cells may have limited ability to process the shRNA into siRNA. Nevertheless, to investigate the effectiveness of the shRNA approach in gene silencing, we used both exogenous luciferase gene and endogenous survivin gene as targets. We showed that either H1 RNA pro-

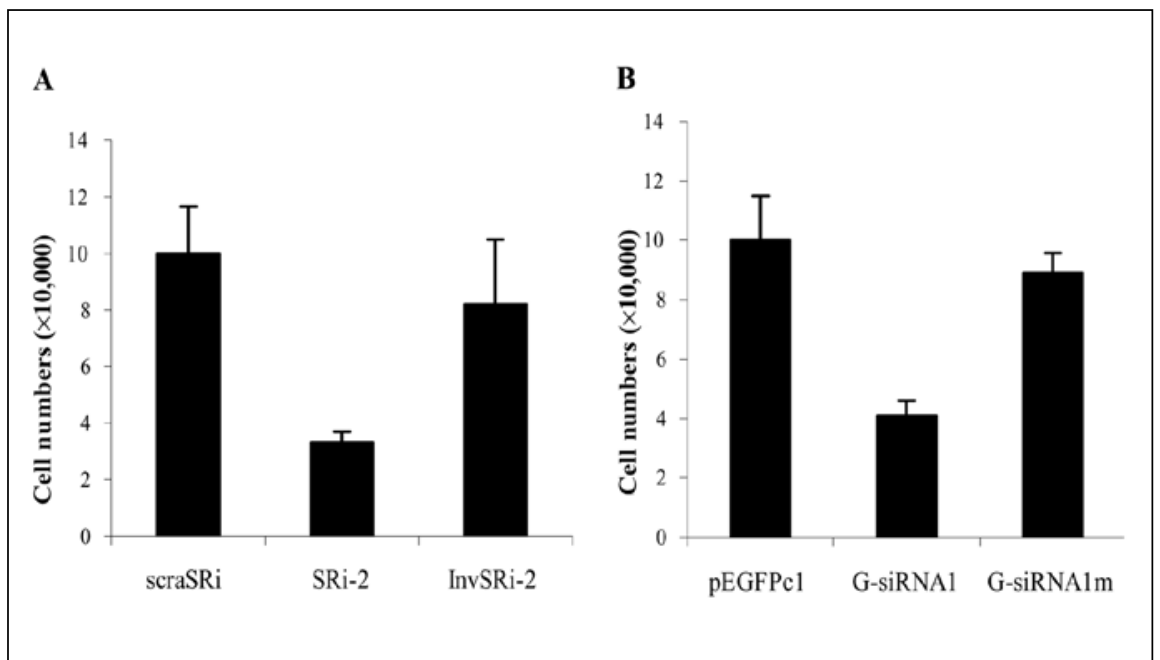

Figure 4. RNAi targeting survivin inhibits cell growth. HeLa cells were transfected with survivin siRNAs (A) or G-shRNA vectors (B). Viable cell numbers were determined by XTT assay $72 \mathrm{~h}$ after transfection. Data from representative experiments in triplicates are shown as the mean $( \pm \mathrm{SD})$. RNAi, RNA interference; siRNA, small interfering RNA; shRNA, short hairpin RNA; XTT, 2,3-bis (2-methoxy-4-nitro-5-sulfophenyl)-2H-tetrazolium-5-carboxanilide.

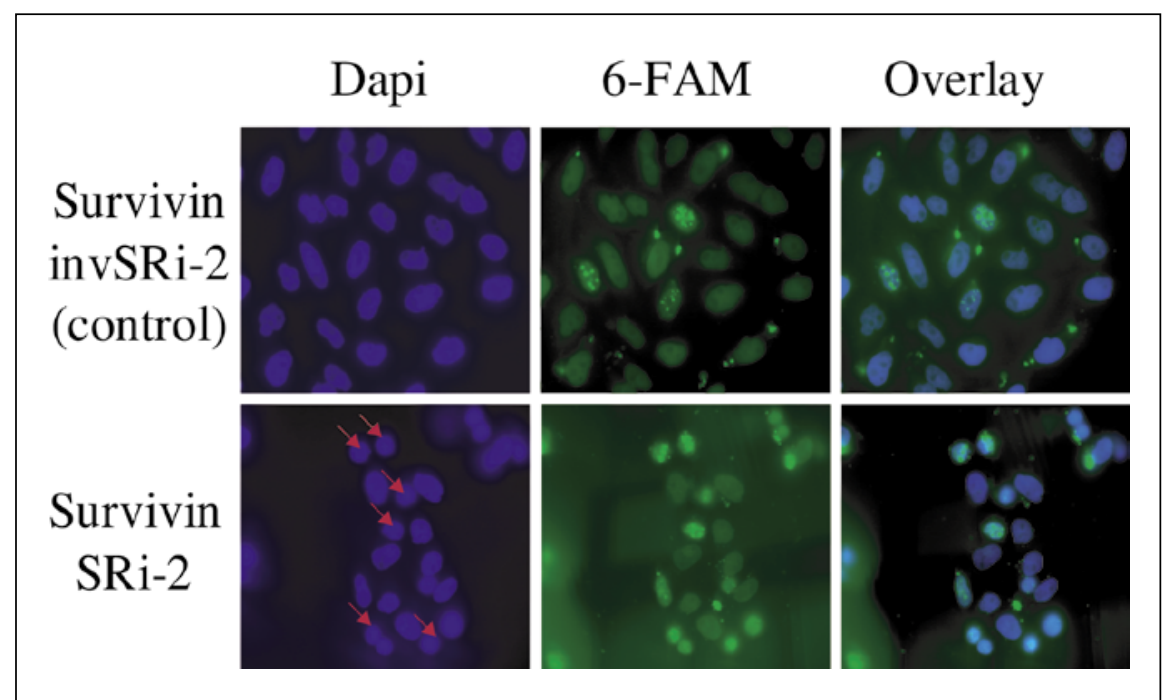

Figure 5. Silencing of survivin expression by RNAi-induced apoptosis. HeLa cells were transfected with 6-FAM fluorescence-labeled survivin siRNA (SRi-2) or control siRNA (InvSRi-2). Cells were fixed and stained with DAPI $48 \mathrm{~h}$ after transfection. The cell morphology was captured under a Zeiss digital fluorescence microscope and processed with Photoshop software. As shown, apoptotic cells possess condensed small nuclei (arrowed). RNAi, RNA interference; siRNA, small interfering RNA. 
moter-mediated or RNA Pol II promoter-mediated expression of shRNA was able to silence gene expression. In addition, we demonstrated that RNAimediated silencing of survivin expression inhibits cell growth and induces apoptosis in the transfected cells.

Here, we would like to point out that, consistent with a recent report that siRNA with mismatched bases in the coding sequence of an endogenous gene can silence gene expression (26), we found that mismatched siRNA or shRNA for survivin or luciferase gene could inhibit targeted gene expression in some degree as well. This observation suggests that mismatch siRNA duplex may be able to suppress expression of off-target cellular genes. Therefore, a careful search for gene database during siRNA or shRNA design may minimize this effect.

In summary, we showed that different approaches of RNAi technology significantly silence the targeted gene expression in cancer cells. We demonstrated for the first time that the RNA Pol II-mediated expression of GFP-shRNA in the untranslated region of enhanced GFP (EGFP) mRNA behind an in frame translation stop codon was able to silence gene expression. This finding may lead to new directions for application of RNAi technology in analysis of the life-essential gene function using many superior mammalian expression vectors.

\section{ACKNOWLEDGMENTS}

This work was supported by the Concern Foundation (Beverly Hills, CA). We cordially thank Drs. Margot Ip and Jayakumar Nair for critical reading of and commenting on this paper.

\section{REFERENCES}

1.Hammond, S.M., A.A. Caudy, and G.J. Hannon. 2001. Post-transcriptional gene silencing by double-stranded RNA. Nat. Rev. Genet. 2:110-119.

2.Bernstein, E., A.M. Denli, and G.J. Hannon. 2001. The rest is silence. RNA 7: 1509-1521.

3.Carthew, R.W. 2001. Gene silencing by double-stranded RNA. Curr. Opin. Cell Biol. 13:244-248.
4.Hannon, G.J. 2002. RNA interference. Nature 418:244-251.

5.McManus, M.T. and P.A. Sharp. 2002. Gene silencing in mammals by small interfering RNAs. Nat. Rev. Genet. 3:737-747.

6.Zamore, P.D., T. Tuschl, P.A. Sharp, and D.P. Bartel. 2000. RNAi: double-stranded RNA directs the ATP-dependent cleavage of mRNA at 21 to 23 nucleotide intervals. Cell 101:25-33.

7.Elbashir, S.M., W. Lendeckel, and T. Tuschl. 2001. RNA interference is mediated by 21- and 22-nucleotide RNAs. Genes Dev. 15: 188-200.

8.Caplen, N.J., J. Fleenor, A. Fire, and R.A. Morgan. 2000. dsRNA-mediated gene silencing in cultured Drosophila cells: a tissue culture model for the analysis of RNA interference. Gene 252:95-105.

9.Ui-Tei, K., S. Zenno, Y. Miyata, and K. Saigo. 2000. Sensitive assay of RNA interference in Drosophila and Chinese hamster cultured cells using firefly luciferase gene as target. FEBS Lett. 479:79-82.

10.Stark, G.R., I.M. Kerr, B.R. Williams, R.H. Silverman, and R.D. Schreiber. 1998. How cells respond to interferons. Annu. Rev. Biochem. 67:227-264.

11.Williams, B.R. 1997. Role of the doublestranded RNA-activated protein kinase (PKR) in cell regulation. Biochem. Soc. Trans. 25:509-513.

12.Elbashir, S.M., J. Harborth, W. Lendeckel, A. Yalcin, K. Weber, and T. Tuschl. 2001. Duplexes of 21-nucleotide RNAs mediate RNA interference in cultured mammalian cells. Nature 411:494-498.

13.Caplen, N.J., S. Parrish, F. Imani, A. Fire, and R.A. Morgan. 2001. Specific inhibition of gene expression by small double-stranded RNAs in invertebrate and vertebrate systems. Proc. Natl. Acad. Sci. USA 98:9742-9747.

14.Reinhart, B.J., F.J. Slack, M. Basson, A.E. Pasquinelli, J.C. Bettinger, A.E. Rougvie, H.R. Horvitz, and G. Ruvkun. 2000. The 21-nucleotide let-7 RNA regulates developmental timing in Caenorhabditis elegans. Nature 403:901-906.

15.Hutvagner, G., J. McLachlan, A.E. Pasquinelli, E. Balint, T. Tuschl, and P.D. Zamore. 2001. A cellular function for the RNA-interference enzyme Dicer in the maturation of the let-7 small temporal RNA. Science 293:834-838.

16.Paddison, P.J., A.A. Caudy, E. Bernstein, G.J. Hannon, and D.S. Conklin. 2002. Short hairpin RNAs (shRNAs) induce sequence-specific silencing in mammalian cells. Genes Dev. 16:948-958.

17.Brummelkamp, T.R., R. Bernards, and R. Agami. 2002. A system for stable expression of short interfering RNAs in mammalian cells. Science 296:550-553.

18.Li, F. 2003. Survivin study: what is the next wave? J. Cell. Physiol. 197:8-29.

19.Li, F., G. Ambrosini, E.Y. Chu, J. Plescia, S. Tognin, P.C. Marchisio, and D.C. Altieri. 1998. Control of apoptosis and mitotic spindle checkpoint by survivin. Nature 396 : 580-584.

20.Ambrosini, G., C. Adida, G. Sirugo, and D.C. Altieri. 1998. Induction of apoptosis and inhibition of cell proliferation by survivin gene targeting. J. Biol. Chem. 273: 11177-11182.

21.Grossman, D., J.M. McNiff, F. Li, and D.C. Altieri. 1999. Expression of the apoptosis inhibitor, survivin, in nonmelanoma skin cancer and gene targeting in a keratinocyte cell line. Lab. Invest. 79:1121-1126.

22.O'Connor, D.S., D. Grossman, J. Plescia, F. Li, H. Zhang, A. Villa, S. Tognin, P.C. Marchisio, and D.C. Altieri. 2000. Regulation of apoptosis at cell division by p34cdc2 phosphorylation of survivin. Proc. Natl. Acad. Sci. USA 97:13103-13107.

23.Olie, R.A., A.P. Simoes-Wust, B. Baumann, S.H. Leech, D. Fabbro, R.A. Stahel, and U. Zangemeister-Wittke. 2000. A novel antisense oligonucleotide targeting survivin expression induces apoptosis and sensitizes lung cancer cells to chemotherapy. Cancer Res. 60:2805-2809.

24.Li, F., E.J. Ackermann, C.F. Bennett, A.L. Rothermel, J. Plescia, S. Tognin, A. Villa, P.C. Marchisio, and D.C. Altieri. 1999. Pleiotropic cell-division defects and apoptosis induced by interference with survivin function. Nat. Cell Biol. 1:461-466.

25.Chen, J., W. Wu, S.K. Tahir, P.E. Kroeger, S.H. Rosenberg, L.M. Cowsert, F. Bennett, S. Krajewski, et al. 2000. Down-regulation of survivin by antisense oligonucleotides increases apoptosis, inhibits cytokinesis and anchorage-independent growth. Neoplasia 2: 235-241.

26.Saxena, S., Z.O. Jonsson, and A. Dutta. 2003. Small RNAs with imperfect match to endogenous mRNA repress translation. Implications for off-target activity of small inhibitory RNA in mammalian cells. J. Biol. Chem. 278:44312-44319.

Received 4 November 2003; accepted 8 December 2003.

\section{Address correspondence to:}

Fengzhi Li

Department of Pharmacology and Therapeutics

Roswell Park Cancer Institute (RPCI)

Elm \& Carlton Streets

Buffalo, NY 14263, USA

e-mail:fengzhi.li@roswellpark.org 